\title{
E.B. Titchener, Lectures on the Elementary Psychology of Feeling and Attention
}

1908

\section{Alain Flajoliet}

\section{(2) OpenEdition}

\section{Journals}

Édition électronique

URL : http://journals.openedition.org/alter/1782

DOI : $10.4000 /$ alter. 1782

ISSN : 2558-7927

Éditeur :

Association ALTER, Archives Husserl (CNRS-UMR 8547)

Édition imprimée

Date de publication : 1 octobre 2010

Pagination : 339-346

ISBN : 2-9522374-6-8

ISSN : 1249-8947

\section{Référence électronique}

Alain Flajoliet, «E.B. Titchener, Lectures on the Elementary Psychology of Feeling and Attention », Alter [En ligne], 18 | 2010, mis en ligne le 01 juin 2020, consulté le 24 septembre 2020. URL : http:// journals.openedition.org/alter/1782 ; DOI : https://doi.org/10.4000/alter.1782 


\title{
E.B. TITCHENER, LECTURES ON THE ELEMENTARY PSYCHOLOGY OF FEELING AND ATTENTION (1908)
}

\author{
Alain Flajoliet
}

Les Conférences sur la psychologie élémentaire du sentiment et de l'attention furent tenues par E.B. Titchener (1867-1927) à l'Université de Columbia en février 1908 et publiées en juillet de la même année à New York (The Macmillan Company). Dans ces conférences, qui traitent successivement de la sensation, du sentiment, et enfin de l'attention, Titchener (1867-1927) fait un point méticuleux de l'état de la question dans la psychologie de l'époque avant d'avancer ses propres hypothèses. Il publia par ailleurs An Outline of Psychology (1896), puis un livre intitulé : Experimental Psychology (1905). La refonte de l'ouvrage de 1896 donna A Text-book of Psychology en deux parties (1909-1910).

Quatre des conférences de 1908 sont consacrées à l'attention, les conférences cinq à huit. La cinquième définit l'attention comme «clarté des sensations» («sensory clearness»). "Je crois (...), explique Titchener, que nous ferions bien de discuter avec le plus grand sérieux d'une psychologie de la clarté, en considérant cette clarté comme un attribut de la sensation, exactement comme la qualité est un attribut de la sensation conditionné par une différenciation des nerfs ». (An Outline, p. 210-211). Les conférences VI et VII dégagent les lois de l'attention. Enfin, l'étude se clôt avec une esquisse d'analyse des rapports entre affection et attention (conférence VIII) sur laquelle nous ne nous attarderons pas. La méthode utilisée dans les pages sur l'attention est celle employée dans le reste de l'ouvrage: rappel de $l^{\prime}$ « état de la question» dans les sciences psychologiques, analyse critique, et proposition de l'interprétation correcte. Parmi les psychologues importants cités dans ces conférences, on trouve W. Wundt (Physiologische Psychologie 1874, 1903), O. Külpe (Grundriss der Psychologie, 1893), Ebbinghaus (Grundzüge der Psychologie, 1905), M. Geiger (Neue Komplikationsversuche, 1903), Münsterberg 


\section{L'attention}

(Grundzüge des Psychologie, 1900), Pillsbury (L'attention, 1906), G.E. Müller (Sinnliche Aufmerksamkeit, 1873).

Avant d'entrer dans le vif du sujet, Titchener rappelle avec force que son étude ne mobilisera que l'introspection, en excluant en particulier ce que la physiologie pourrait expliquer de l'intensification de telle ou telle sensation en fonction de processus nerveux sous-jacents. (Nous verrons que cette exclusion est loin d'être toujours rigoureuse).

\section{L'attention comme clarté sensorielle}

Titchener se place dans le sillage de la Physiologische Psychologie de Wundt $(1874,1903)$. En 1874, ce dernier avait ouvert son étude de l'attention par la distinction (appelée à un bel) avenir entre le « champ visuel » (Blickfeld) et le «point de fixation du regard» (Blickpunkt). En 1903, prolongeant cette intuition, il définissait l'attention comme une redistribution de la clarté dans la conscience : elle augmente pour certaines représentations en s'accompagnant $d^{\prime}$ un sentiment d'activité, elle diminue pour d'autres en s'accompagnant d'un sentiment d'inhibition. C'est aussi le point de vue d'Ebbinghaus (Grundzüge der Psychologie) et de Pillsbury (L'attention), qui ont tout comme Wundt les faveurs de Titchener. La première question qui se pose dès lors est celle des conditions de cette augmentation de clarté sensorielle par laquelle on vient de définir l'attention. On peut selon Titchener en envisager huit nous allons vérifier que dans leur examen il abandonne parfois la stricte méthode introspective pour des considérations psycho-physiologiques.

a) L'intensité $d u$ stimulus. Elle n'est autre que ce qui constitue l'origine même des sensations sur lesquelles se porte l'attention.

b) La forme et la qualité du stimulus. Nous ne parvenons pas, par exemple, à détacher notre attention d'une douleur peu intense mais qui est ressentie comme insistante, importune, pressante, etc. G.E. Müller a bien saisi ce phénomène lorsqu'il a affirmé que la «qualité » (Qualität) du stimulus conditionnait son « insistance» (Eindringlichkeit).

c) La répétition conditionne la clarté de nos sensations parce qu'elle a le pouvoir d'éveiller progressivement notre attention par la sommation des stimuli.

d) Le mouvement du stimulus en fait plus aisément le centre de notre attention. Stumpf a fait une observation qui va en ce sens. Supposons l'ombre immobile d'un crayon immobile sur une feuille de papier blanc et qu'on ne remarque pas. Elle sera immédiatement remarquée si l'on fait bouger le crayon et, avec lui, son ombre.

e) La nouveauté, la rareté, l'étrangeté du stimulus, bref le fait de n'y être pas habitué, sont des traits qui contribuent à frapper l'attention, comme le soulignent tant G.E. Müller que James, Külpe, Ebbinghaus, Pillsbury. Tout en acceptant cette analyse, Titchener apporte une précision intéressante. Un 
stimulus nouveau et inhabituel, dit-il, est un stimulus non encore inscrit dans les chaînes associatives de l'esprit, donc isolé.

f) Les connexions associatives. Une sensation est d'autant plus remarquée que le sujet peut se préparer à l'accueillir par l'existence dans son esprit de connexions associatives où les sensations ont déjà été intégrées par l'habitude (Müller et Ebbinghaus). Helmholtz a fait des expériences sur la vision stéréoscopique et sur l'audition de sons confirmant cette hypothèse. On peut remarquer d'autant plus, dit-il, les deux images composant la vision stéréoscopique ou les sons composant un accord que l'on s'est préalablement entraîné à se représenter concrètement comment ces images ou ce son devaient apparaître.

g) L'accommodation des organes des sens. Pour Wundt par exemple, la fixation est l'une des conditions externes de la clarté visuelle. Sur ce point, Külpe est plus réservé. "Nous serons plus exacts, affirme-t-il, en supposant que ces [conditions motrices] ne sont qu'indirectement favorables à l'aperception de contenus particuliers en tant qu'elles déterminent les attributs des contenus eux-mêmes ». Sub audiendo: l'accommodation n'est pas une vraie condition de l'aperception, c'est-à-dire de l'attention, elle ne fait que favoriser cette dernière. Toutefois, Titchener n'est pas convaincu par ces réserves de Külpe. On peut parfaitement faire de l'accommodation la vraie condition de l'apparition dans les contenus sensibles de l'attribut de la clarté, bref de l'attention, ne serait-ce que pour cette raison: si l'accommodation sensorielle n'est pas faite, l'attention ne suit pas.

h) L'absence ou la disparition d'un stimulus attire l'attention. Fechner donne l'exemple du meunier qui ne s'aperçoit du bruit de son moulin que lorsque ce dernier s'arrête inopinément. Titchener propose de distinguer deux cas bien distincts. Soit le stimulus en question avait déjà été remarqué (nous nous attendons à ce que le tic-tac régulier de l'horloge se poursuive tout au long de la journée), soit il s'agit d'un stimulus non remarqué (des fleurs tout à fait nouvelles sont posées sur la cheminée). Dans le second cas, la disparition du stimulus (les fleurs sont ôtées de la cheminée) n'est pas remarquée.

\section{Les lois de l'attention}

Titchener en examine sept.

a) La clarté des sensations peut, dans certaines limites, varier indépendamment des autres attributs des sensations et en particulier de l'intensité. Cette loi fait débat et Titchener évoque à ce propos une anecdote relatée par Stumpf dans sa Tonpsychologie. Pour faire une expérience introspective validant ou infirmant cette hypothèse, Stumpf et Mach s'assirent devant un harmonium et se firent jouer un accord tenu avec pour consigne d'abord de focaliser leur attention sur l'accord puis un seul son; il fallait dire s'ils ressentaient simultanément une augmentation en intensité du son ainsi isolé. Problème : 


\section{L'attention}

Mach affirma qu'il avait parfaitement perçu l'intensification du son, tandis que Stumpf, malgré tous ses efforts, avoua n'avoir rien perçu du tout. De fait, dans le champ psychologique du début du siècle, les avis divergent. Peu de psychologues de l'époque n'ont pas pris parti dans la querelle. Pour Wundt, si la clarté et la force d'une impression sont deux choses bien différentes, cela n'implique pas qu'elles ne puissent avoir une certaine influence l'une sur l'autre - c'est ce qu'il admet lorsqu'il dit qu'au moment où on remarque le son de l'horloge d'un clocher, ce son devient pour notre perception à la fois plus clair et plus intense. Dans l'ensemble, l'orientation qui commence à dominer à l'époque des Lectures de Titchener va dans le sens d'une corrélation entre attention et intensité des sensations, au moins dans certaines limites. Külpe affirme en ce sens (Grundriss der Psychologie) $\mathrm{qu}^{\prime}$ « à l'intérieur de certaines limites étroites (...) les contenus sont réellement intensifiés dans l'état d'attention ». Ebbinghaus se range dans le camp de Wundt et de Külpe. Münsterberg en revanche (Grundzüge der Psychologie) conteste vigoureusement toute possibilité soit d'affaiblir l'intensité d'une sensation en diminuant l'attention qu'on lui porte, soit d'augmenter cette intensité en lui portant une attention plus soutenue. Titchener s'appuie sur certaines expériences menées à l'université de Cornell par Bentley pour valider l'hypothèse des variations corrélatives de l'attention et de l'intensité des sensations.

b) La loi des niveaux ou des degrés de clarté de l'attention. Observons d'abord que cette loi n'est pas acceptée par tous les psychologues. Külpe notamment récuse ce concept de degrés de clarté dans la conscience et opte pour l'hypothèse selon laquelle des distinctions très tranchées de clarté, voire carrément des sauts d'une clarté maximale à l'obscurité extrême affectent les contenus de conscience. Mais Titchener observe que ses arguments sont fragilisés par une certaine confusion entre ce qui relève de la physiologie et de la psychologie introspective. Opposés à Külpe, les tenants de l'existence de degrés de l'attention sont d'importants psychologues comme J. Ward, Helmholtz, Wundt, Baldwin.

La loi des degrés de l'attention affirme d'abord que la clarté dans les contenus de conscience connaît des degrés, depuis une zone d'attention maximale jusqu'à une zone obscure où aucun contenu n'est remarqué. Ensuite, cette loi dénombre et décrit les zones (claire, demi-claire, obscure...), mais avec des divergences selon les psychologues. Baldwin défend l'hypothèse (Sense and Intellect, 1890) selon laquelle ces zones de la conscience claire sont au nombre de quatre. En allant de la plus claire à la plus obscure: $l^{\prime}$ « aperception », la «conscience attentive », la "conscience diffuse », le "subconscient. Wundt et Helmholtz (Zur Lehre von den Tonempfindungen, 1877) distinguent, pour toute conscience attentive, deux types de rapport de la conscience à ses contenus. Soit les contenus, explique Helmholtz en se référant à Leibniz, sont clairs et donnés dans une « aperception » (Apperception), soit ils ne sont pas clairs et sont seulement donnés dans une "perception » (Perception). Et Wundt de son côté distingue dans la conscience visuelle, 
nous l'avons vu, le point de fixation clair (Blickpunkt) de son attention et le champ visuel (Blickfeld) non clair sur lequel s'enlève la focalisation de l'attention. Voici comment il s'exprime dans sa Physiologische Psychologie. "Si nous disons des représentations présentes dans un moment donné qu'elles sont dans le champ visuel, on peut caractériser cette partie du champ vers laquelle l'attention est tournée comme le point de fixation interne (innere Blickpunkt) (...). Le point de fixation interne peut se déplacer successivement vers les différentes parties du champ visuel interne (des inneren Blickfeldes) ». Bien que Wundt soit souvent considéré comme l'inventeur de cette métaphore fameuse, il l'a très probablement selon Titchener empruntée à K. Fortlage, qui lui-même la tenait de Lotze. Quoi qu'il en soit, elle revient à défendre l'existence de deux zones dans les contenus de la conscience attentive, la zone claire au point de fixation et la zone obscure du champ.

Ayant passé en revue tous ces travaux de Ward, Wundt, Baldwin, etc., Titchener en fait un bilan critique. Il existe encore trop de divergences et d'imprécisions dans les descriptions des zones et des degrés de clarté de la conscience chez tous ces psychologues. L'essentiel du travail scientifique reste donc à faire, soit du côté de la conscience claire, soit du côté de la conscience obscure. Beaucoup de questions, écrit-il, restent pour le moment en suspens. Dans la zone de la conscience non claire, trouve-t-on certaines différences d'obscurité, ou bien l'obscurité est-elle pour ainsi dire homogène? Mais comment pratiquer l'introspection dans cette zone par définition obscure?

La chose est plus facile du côté des contenus clairs et Titchener affirme qu'au niveau de la conscience claire il existe des différenciations significatives dont la description est une tâche importante pour la psychologie. Il résume certains travaux encourageants de Dietze (Untersuchungen über den Umfang des Bewussteins, Philosophische Studien, 1885) et de Wundt sur l' "étendue» (Umfang) de l'attention. Dietze a fait des expériences dans lesquelles le sujet écoute des séries de battements d'un métronome, qui sont séparées par la sonnerie d'une cloche. Il a observé que tout sujet prenant conscience d'une série lors de la sonnerie perçoit un dégradé de clarté, d'une part par rapport au moment présent et d'autre part par rapport au point focal de son attention. Titchener résume: les représentations possèdent un degré de clarté qui est pour une part fonction du temps écoulé et pour une autre part fonction de l'importance de l'accentuation subjective. Wundt de son côté a dégagé l'idée que dans le champ visuel, on peut distinguer une zone claire, une zone demi-claire, et une zone tout à fait obscure. Voici la conclusion principale de Titchener: il existe des différences remarquables de clarté au niveau supérieur de la conscience, et le laboratoire de l'Université de Leipzig est en train de travailler à les étudier avec succès. La conscience supérieure n'est pas homogène, on y trouve toutes une série de nuances qui vont du très clair au semi-obscur. Ces nuances peuvent et doivent être décrites introspectivement.

c) Les lois de l'accommodation et de l'inertie. 


\section{L'attention}

Stumpf donne cette définition de la loi de l'accommodation dans sa Tonpsychologie: "L'attention a besoin d'un certain temps pour s'accommoder pour ainsi dire à l'impression, pour atteindre son maximum ». Ce ne sont donc pas seulement les sens qui doivent s'accommoder aux stimuli, mais aussi d'une certaine façon l'attention que l'on porte aux sensations (provoquées dans les organes par les stimuli) qui doit s'adapter aux sensations. L'accommodation sensorielle précède l'éventuelle accommodation de l'attention, la seconde pouvant n'être pas là lorsque la première est là. Comme toute accommodation, l'accommodation attentionnelle prend un certain temps qui diminue par apprentissage.

La loi d'inertie ne pose guère de problèmes. Fechner la formule ainsi (Revision der Hauptpunkte des Psychophysik, 1882) : «Il nous convient mieux, jusqu'à un certain point, de demeurer dans une direction - et par là dans une activité - de l'attention déjà tenue, que de les abandonner et de cesser l'activité ".

d) La loi de l'entrée prioritaire (prior entry). Elle est issue d'un article fondamental de M. Geiger (Neue Komplikationsversuche, 1903). L'expérience est la suivante. Une aiguille tourne à une vitesse uniforme devant le cadran gradué d'une horloge. A un moment de la révolution (inconnu du sujet), une cloche sonne. Le sujet doit annoncer le moment de coïncidence subjective entre la perception visuelle et la perception sonore (moment où se forme une « complication » au sens herbartien). Il est donc obligé (c'est le point décisif) de concentrer son attention sur la venue du son. On observe un résultat général important et curieux : sous la condition d'une forte attention portée au son, ce dernier semble subir un déplacement négatif sur le cadran; pour la conscience, il est joint à une gradation de l'horloge que l'aiguille a déjà dépassé quand le son objectif est produit. Le son est en quelque sorte (pour l'introspection) repoussé en arrière, on l'entend "trop tôt». Pourquoi cette étrange erreur subjective d'appréciation? L'explication, très difficile, a mobilisé les efforts de nombreux psychologues et c'est M. Geiger qui en a fourni lui-même la plus convaincante.

Il explique ainsi (Physiologische Psychologie, 1903) le résultat de son expérience. L'impression subjective que le son se lie avec une gradation du cadran exprimant un instant antérieur à celui que l'aiguille désigne (alors qu'objectivement il devrait y avoir coïncidence entre le son, la place de l'aiguille et le moment temporel) est un effet de l'attention portée au stimulus sonore. D'autant plus, dit-il, l'attention est concentrée sur l'impression sonore au détriment de l'impression visuelle de la flèche, d'autant plus l'impression visuelle se déplace au début du laps de temps du sens de la vue qui lui est ordonné. Dans la situation de l'expérience, concentrer son attention sur le stimulus sonore lui fait prendre subjectivement le pas sur le stimulus visuel, et il cesse alors de coïncider parfaitement avec l'instant désigné sur le cadran par la flèche : il semble apparaître avant ce dernier (c'est-à-dire coïncider avec une graduation antérieure du cadran). On peut faire aussi l'expérience symétrique et inverse. Plus la concentration, affirme Geiger, est rendue difficile ou diminue pour une raison quelconque, plus l'impression sonore est repoussée 
vers la fin du laps de temps. Ici, l'impression visuelle de la flèche prend le pas sur l'impression sonore parce que cette dernière n'est plus soutenue par l'attention : pour le sujet le son semble alors apparaître après l'instant désigné par la flèche (c'est-à-dire coïncider avec une graduation du cadran plus éloignée vers l'avant, selon un déplacement cette fois-ci positif).

e) La loi de l'étendue limitée de l'attention. Les faits sont simples, et l'interprétation de Wundt correcte selon Titchener. Si un groupe d'objets (lignes, figures géométriques, nombres, lettres, mots courts) qui se tiennent à intérieur du champ de vision claire sont momentanément exposés au moyen d'un tachistoscope, l'observateur averti en reconnaîtra tout au plus cinq ou six dans un seul acte d'attention. Il y a donc une limite à la capacité de saisie de l'attention visuelle.

f) La loi de l'instabilité temporelle de l'attention. Titchener commence par rappeler une loi formulée par Wundt, qui lui paraît incorrecte. Ce dernier affirme dans sa Physiologische Psychologie: «(...) Entre l'aperception (Apperception) de deux représentations successives il $\mathrm{y}$ a toujours un intervalle de temps dans lequel, pour être clairement aperçues, la première a déjà sombré trop loin, et l'autre ne s'est pas suffisamment exhaussée. » Autrement dit, si l'attention saisit deux représentations claires l'une après l'autre, il y a un intervalle d'obscurité entre les deux et elle est donc intermittente. Titchener ne trouve pas que l'introspection justifie cette notion $\mathrm{d}^{\prime}$ « intermittence » de l'attention.

En revanche, il existe bien un phénomène qui mérite analyse, celui des «fluctuations de l'attention » (Apperceptionswellen, fluctations of attention). Deux questions essentielles sont en suspens. D'abord, est-ce que la fluctuation s'observe dans tous les domaines sensoriels ? La question fait débat. Par exemple, dans le domaine du toucher, Lange, Lehmann et Wiersma penchent pour l'existence de fluctuations, ce que contestent Ferree et Geissler. Titchener ne prend pas parti. La seconde question porte sur les conditions de ces fluctuations: sont-elles centrales ou périphériques? Titchener donne la préférence à la conception de Ferree, qui fait jouer un rôle décisif aux conditions périphériques des fluctuations. "En elle-même, explique Ferree, l'attention est un phénomène continu, mais sa continuité est gênée par les mouvements oculaires, les clignements d'yeux, etc. En raison de ces influences, probablement essentiellement en raison de celle du seul mouvement oculaire, elle devient un processus intermittent (...)».

g) La loi du degré de clarté de l'attention. C'est le souhait des psychologues (par exemple Külpe) de formuler une loi qui serait à la clarté ce que la loi de Weber est à l'intensité de la sensation. Mais comment «mesurer l'attention »? Deux voies s'ouvrent, l'une directe et l'autre indirecte, mais aucune n'a donné de résultats totalement satisfaisants. La voie directe est la voie introspective. Pour réussir, elle supposerait la satisfaction de conditions très contraignantes. Supposons un sujet ayant acquis la sensibilité discriminative maximale et supposons constante cette sensibilité. Supposons en outre une série de stimuli capables de le distraire, homogènes en genre mais présentant 


\section{L'attention}

une gradation dans la complexité, de telle sorte que l'on puisse réduire le pourcentage de réponses correctes du sujet de 100 à 95 , de 96 à 85, etc. en fonction du « degré de distraction » produit. L'action des facteurs qui distraient est supposée constante ; et il est nécessaire qu'ils soient du même genre et donc exercent une influence qui diffère seulement, et de façon mesurable, en degré. Dans ces conditions le sujet doit décider si la clarté des contenus était différente pour sa perception lorsque la distraction était de $5 \%$ et de $10 \%$, de 5 et de $15 \%$, de 80 et de $85 \%$, etc. On obtient alors en principe une "échelle de clarté » selon les différences sensibles, parallèle à l'échelle des quantités mesurées de stimuli qui distraient. Titchener explique alors que les tentatives menées avec ses étudiants pour mettre en œuvre ce protocole ont pour l'instant échoué.

L'autre voie (indirecte) offre aussi de grosses difficultés. Il s'agirait de mesurer l'attention indirectement par les sensations de tension qui l'accompagnent. C'est la voie proposée par Stumpf. Le problème non résolu est double. $\mathrm{D}^{\prime}$ abord les différents degrés de la sensation d'effort ne sont pas parallèles aux degrés de clarté. L'effort concomitant n'indique pas un état d'attention, mais plutôt une gêne de l'attention ne parvenant pas à s'établir. En même temps, il n'est pas non plus une mesure inverse du degré de clarté : sous certaines conditions, un léger effort (pour contrer une petite distraction) est favorable à l'attention.

Titchener conclut: il y a encore beaucoup de travail à accomplir pour inventer les tests objectifs qui permettront de se rapprocher d'une mesure rigoureuse de l'attention.

$\mathrm{Au}$ total, les Lectures de Titchener sont un outil extrêmement précieux pour l'historien de la psychologie qui serait à la recherche d'une synthèse juste et précise des principaux travaux en psychologie de l'attention au tournant du siècle. On n'y trouve pas en revanche de véritable théorie systématisée de l'attention, mais plutôt un tri au cas par cas entre hypothèses valides et hypothèses contestables. 